\title{
Privacy-by-Design Task Offloading for UAV-mounted Cloudlets
}

\author{
Weibin Ma \\ Department of Computer and Information Sciences \\ University of Delaware \\ Newark, DE, USA \\ weibinma@udel.edu
}

\author{
Lena Mashayekhy \\ Department of Computer and Information Sciences \\ University of Delaware \\ Newark, DE, USA \\ mlena@udel.edu
}

\begin{abstract}
We consider a network of capacitated Unmanned Aerial Vehicles (UAVs) cooperating as an aerial edge computing system. Due to limited onboard energy and computation capabilities of UAV-mounted cloudlets, a single UAV might not be able to execute a large number of tasks and guarantee their desired quality of services. The overloaded UAV can fulfill its overwhelming workload by offloading its tasks to other UAVs. However, data privacy and accessibility are of critical importance that need to be considered for offloading. We propose a privacy-by-design offloading solution for an overloaded UAV aiming to minimize the offloading cost while satisfying the quality of services.
\end{abstract}

\section{KEYWORDS}

Edge Computing, Unmanned Aerial Vehicles, Data Protection

\section{ACM Reference Format:}

Weibin Ma and Lena Mashayekhy. 2019. Privacy-by-Design Task Offloading for UAV-mounted Cloudlets. In The Fourth ACM/IEEE Symposium on Edge Computing (SEC 2019), November 7-9, 2019, Arlington, VA, USA. ACM, New York, NY, USA, 3 pages. https://doi.org/10.1145/3318216.3363320

\section{INTRODUCTION}

In mobile edge computing, deploying cloudlet infrastructure at the edge of the network is costly and may not be feasible in many situations (e.g., disaster situations, emergency rescue, unexpected surge in user demand) and regions with sparse or no infrastructure of wireless access points such as remote rural areas. To overcome these problems, considering Unmanned Aerial Vehicles (UAVs) as computational cloudlets is proposed by virtue of their inherent attributes such as aerial mobility, low operating costs, flexible deployment, and wireless communication ability $[2,8,9]$. UAV-mounted cloudlets can provide edge computing services and hence improve quality-of-service (QoS) for ground IoTs [3, 11].

While UAV-mounted cloudlets can bring many opportunities to avoid QoS violations and balance the load, they have often limited computing capacities and energy budgets. To expand the computational capacity and coverage of a single UAV-mounted cloudlet, especially when serving large computational demands, autonomous cooperation and coordination among multiple UAVs are needed

Permission to make digital or hard copies of all or part of this work for personal or classroom use is granted without fee provided that copies are not made or distributed for profit or commercial advantage and that copies bear this notice and the full citation on the first page. Copyrights for components of this work owned by others than ACM must be honored. Abstracting with credit is permitted. To copy otherwise, or republish, to post on servers or to redistribute to lists, requires prior specific permission and/or a fee. Request permissions from permissions@acm.org.

SEC 2019, November 7-9, 2019, Arlington, VA, USA

(C) 2019 Association for Computing Machinery.

ACM ISBN 978-1-4503-6733-2/19/11 . .\$15.00

https://doi.org/10.1145/3318216.3363320 to form an interconnected computing system and improve quality of edge services $[4,5]$. Such a perspective, however, opens new research challenges on account of the current lack of efficient data protection mechanisms for offloading. In particular, the major categories are considered privacy and accessibility restrictions defined as follows: 1) Privacy restrictions: If some tasks are offloaded to the same UAV (i.e., cloudlet) for computing, they can reveal sensitive information, which could harm IoT user privacy [7, 10]; 2) Accessibility restrictions: There are often some restrictions in offloading a task to a specific UAV due to lack of trust, reliability, or system compatibility/preferences $[6,12]$.

In this paper, we consider a network of capacitated UAV-mounted cloudlets (NUMC) cooperating as an aerial computing system and propose a privacy-by-design offloading solution for an overloaded UAV aiming to minimize offloading cost while satisfying quality of services and handling both restrictions. We first formulate an optimal offloading mathematical model for this Data pRotection Offloading Problem (DROP). DROP is an NP-hard problem. We then propose an iterative algorithm by exploiting graph theory to obtain efficient and computationally tractable solutions. We finally evaluate the performance of our proposed algorithm in extensive experiments.

\section{OPTIMIZATION FORMATION}

We define $u_{0}$ as an overloaded UAV and $U=\left\{u_{1}, \ldots, u_{m}\right\}$ as a set of $m$ UAVs with available computational resources. The set of tasks at $u_{0}$ to be offloaded is defined as $K=\left\{k_{1}, \ldots, k_{n}\right\}$, where $n$ is the number of tasks and $n \geq 1$ ( $u_{0}$ has at least one task to be offloaded). Let $F_{i}$ be the CPU frequency (i.e., CPU cycles per second) of $u_{i}$, and its capacity is denoted by $u_{i}^{c a p}=\left(C_{i}^{c a p}, D_{i}^{c a p}\right)$ where $C_{i}^{c a p}$ is the number of idle computational cycles of $u_{i}$ and $D_{i}^{c a p}$ is the available memory size of $u_{i}$. Each task $k_{i} \in K$ has some requirements defined by $\left(C_{i}, D_{i}\right)$, where $C_{i}$ represents the number of computational cycles required to obtain the outcome of $k_{i}$, and $D_{i}$ denotes the data size of $k_{i}$.

The total energy consumption for completing $k_{i}$ via offloading to $u_{j} \in U$ can be computed by [5]:

$$
E_{j}(i)=\frac{D_{i} P_{0}}{B_{j}}+C_{i} \gamma\left(F_{j}\right)^{2}
$$

where $P_{0}$ denotes the transmission power of $u_{0}, \gamma$ is the effective switched capacitance (input transition), and $B_{j}$ represents the bandwidth of $u_{j}$.

Similarly, the total delay of completing task $k_{i}$ by offloading to $u_{j}$ is calculated by:

$$
T_{j}(i)=\frac{D_{i}}{B_{j}}+\frac{C_{i}}{F_{j}} .
$$




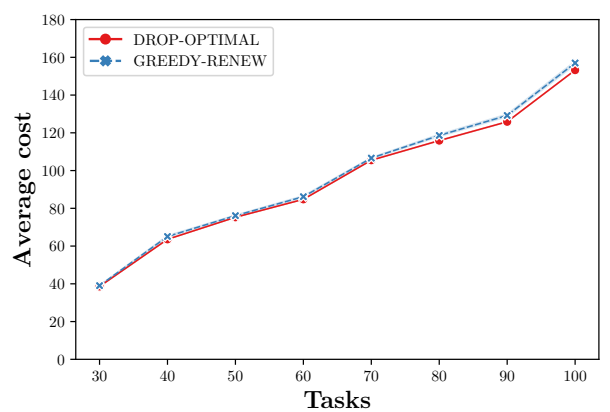

Figure 1: Average cost

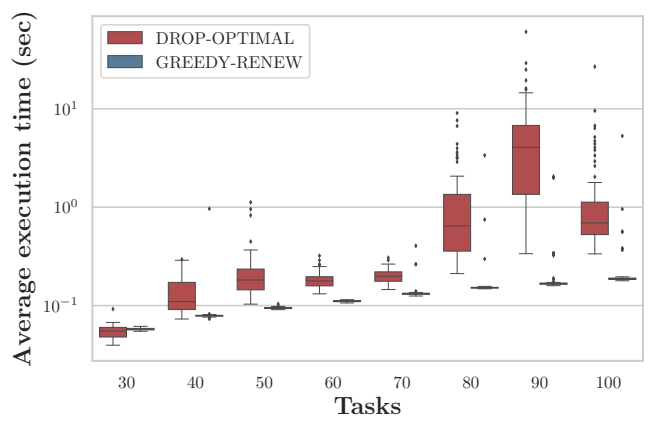

Figure 2: Average execution time
Therefore, we define the overhead cost of offloading $k_{i}$ to $u_{j}$, in terms of energy consumption and processing time, as:

$$
Z_{j}(i)=\alpha_{i} E_{j}(i)+\beta_{i} T_{j}(i),
$$

where $0 \leq \alpha_{i}, \beta_{i} \leq 1$ represent the relative importance weights of energy consumption and processing time for task $k_{i}$ according to its required QoS and current battery lifetime of UAVs. This provides a rich modeling flexibility that fits the general case, where each task may have different importance weight values.

We now formulate the data protection offloading problem (DROP) as an Integer Program (IP) as follows:

$$
\operatorname{Minimize} \sum_{i=1}^{n} \sum_{j=1}^{m} Z_{j}(i) x_{i j}
$$

Subject to:

$$
\begin{array}{lr}
\sum_{i=1}^{n} C_{i} x_{i j} \leq C_{j}^{c a p}, & \forall j \in\{1, \ldots, m\}, \\
\sum_{i=1}^{n} D_{i} x_{i j} \leq D_{j}^{c a p}, & \forall j \in\{1, \ldots, m\}, \\
\sum_{j=1}^{m} b_{i j} x_{i j}=1, & \forall i \in\{1, \ldots, n\}, \\
x_{u j}+x_{v j} \leq 1, & \forall\langle u, v\rangle \in E, \forall j \in\{1, \ldots, m\}, \\
x_{i j} \in\{0,1\}, & \forall i \in\{1, \ldots, n\}, \forall j \in\{1, \ldots, m\},
\end{array}
$$

where $x_{i j}$ is a decision variable with a value of 1 if $k_{i}$ is offloaded to $u_{j}$ and 0 otherwise. To formulate the accessibility restriction, we define an indicator variable, $b_{i j}$. If $k_{i}$ can be offloaded to $u_{j}$ then $b_{i j}=1$, otherwise $b_{i j}=0$. To formulate the privacy restriction, we define $E$ consisting a pair of conflicting tasks that cannot be offloaded to same UAV. The objective function (4) is to minimize the overhead cost for all tasks. Constraints (5) and (6) ensure that the assignment of tasks to each resource-rich UAV does not exceed the capacity of that UAV. Constraints (7) ensure that each task is offloaded to exactly one UAV obeying the accessibility restrictions. Constraints (8) ensure that any two tasks with privacy restrictions cannot be offloaded to the same UAV. Constraints (9) guarantee that the decision variables are binary.

\section{GREEDY-RENEW ALGORITHM}

Due to the NP-hardness of the DROP, we propose an efficient iterative approach, called GREEDY-RENEW.

We first design an UAV Selection Scheme (USS) aiming to decide which task should be selected for offloading at each iteration. USS finds the tasks with minimum number of offloading choices (accessibility). Specifically, if only one task exists, USS selects that task. Otherwise, it selects a task with minimum degree (number of privacy conflicts). After obtaining the target task by USS, we obtain its offloading UAV that has the minimum overhead cost (this step will be enhanced by our renew rule). This procedure continues iteratively.

With defining these priorities for offloading, USS can largely reduce the chance of finding infeasible solutions. However, it is still difficult to find a feasible solution for DROP because of being NPhard. To boost the probability of finding a complete solution, where all tasks are offloaded while satisfying the required restrictions and constraints, we then design a renew rule. In doing so, we first sort all accessible UAVs for task $k_{i}$, in ascending order of their cost, denoted by $L^{s}\left(k_{i}\right)$. Then, the first UAV in $L^{s}\left(k_{i}\right)$ will be selected for the offloading of task $k_{i}$. If a complete assignment is not obtained by the USS, renew rule updates the offloading decision of task $k_{i}$ to the next UAV in $L^{s}\left(k_{i}\right)$. After checking all accessible UAVs of a task if the renew rule cannot find a complete assignment for the task, it halts and moves to the next task. Note that the renew rule only applies when USS cannot achieve a complete assignment.

\section{NUMERICAL EXPERIMENTS}

For privacy restrictions, we setup a well-known random graph model, the Erdös-Rényi model $(N, P)[1]$, where $N$ is the number of tasks and $P$ is probability of having a conflict with another task. For accessibility restrictions, the probability associated with UAVs for each task is $P_{01}$ (the probability of generating 1 using the Bernoulli distribution). Fig. 1 shows that our proposed algorithm obtains near optimal solution. Fig. 2 presents that the proposed algorithm scales well with various number of tasks.

\section{CONCLUSION}

In this paper, we formulated a general data protection offloading problem (DROP) for offloading tasks among a swarm of UAVs. We 
proposed a highly scalable privacy-by-design iterative algorithm that finds near-optimal solutions.

\section{ACKNOWLEDGMENTS}

This research was supported in part by NSF grant CNS-1755913.

\section{REFERENCES}

[1] P Erdös and A Rényi. 1959. On random graphs. Publ. Math 6 (1959), 290-297.

[2] Seongah Jeong, Osvaldo Simeone, and Joonhyuk Kang. 2017. Mobile edge computing via a UAV-mounted cloudlet: Optimization of bit allocation and path planning. IEEE Transactions on Vehicular Technology 67, 3 (2017), 2049-2063.

[3] Shaoshan Liu, Liangkai Liu, Jie Tang, Bo Yu, Yifan Wang, and Weisong Shi. 2019. Edge Computing for Autonomous Driving: Opportunities and Challenges. Proc. IEEE (2019).

[4] Feng Luo, Chunxiao Jiang, Shui Yu, Jingjing Wang, Yipeng Li, and Yong Ren 2017. Stability of cloud-based UAV systems supporting big data acquisition and processing. IEEE Transactions on Cloud Computing (2017).

[5] Weibin Ma, Xuanzhang Liu, and Lena Mashayekhy. 2019. A Strategic Game for Task Offloading among Capacitated UAV-mounted Cloudlets. In Proceedings of the IEEE International Congress on Internet of Things. 1-8.

[6] Lena Mashayekhy, Mark Nejad, and Daniel Grosu. 2019. A Trust-Aware Mechanism for Cloud Federation Formation. IEEE Transactions on Cloud Computing (in press) (2019).

[7] Lena Mashayekhy, Mahyar Movahed Nejad, and Daniel Grosu. 2014. A framework for data protection in cloud federations. In Proceedings of the 43rd International Conference on Parallel Processing. 283-290.

[8] Mohamed-Ayoub Messous, Hichem Sedjelmaci, Noureddin Houari, and SidiMohammed Senouci. 2017. Computation offloading game for an UAV network in mobile edge computing. In Proceedings of the IEEE International Conference on Communications (ICC). 1-6.

[9] Mohammad Mozaffari, Walid Saad, Mehdi Bennis, and Mérouane Debbah. 2016. Unmanned aerial vehicle with underlaid device-to-device communications: Performance and tradeoffs. IEEE Transactions on Wireless Communications 15, 6 (2016), 3949-3963.

[10] Siani Pearson. 2009. Taking account of privacy when designing cloud computing services. In Proceedings of ICSE Workshop on Software Engineering Challenges of Cloud Computing. IEEE, 44-52.

[11] Nafiseh Sharghivand, Farnaz Derakhshan, and Lena Mashayekhy. 2018. QoSAware Matching of Edge Computing Services to Internet of Things. In Proceedings of the 37th IEEE International Performance Computing and Communications Conference. 1-8.

[12] Daniel Zhang, Yue Ma, Chao Zheng, Yang Zhang, X Sharon Hu, and Dong Wang. 2018. Cooperative-competitive task allocation in edge computing for delaysensitive social sensing. In Proceedings of the IEEE/ACM Symposium on Edge Computing (SEC). 243-259. 